\title{
Indentation and Transverse Diameter of the Meckel Cave: Imaging Markers to Diagnose Idiopathic Intracranial Hypertension
}

\author{
(D) A. Kamali, (D).C. Sullivan, (DF. Rahmani, (D)A. Gandhi, (D)A. Aein, (D). Arevalo, (DP. Rabiei, (D).J. Choi, \\ (D) X. Zhang, (D).E. Gabr, and (D)R.F. Riascos
}

\begin{abstract}
BACKGROUND AND PURPOSE: Clinical and imaging manifestations of idiopathic intracranial hypertension should prompt early diagnosis and treatment to avoid complications. Multiple diagnostic imaging criteria are reported to suggest the diagnosis of idiopathic intracranial hypertension with questionable sensitivity and/or specificity. Increased intracranial pressure results in dilation of the perineural cisternal spaces such as the optic nerve sheaths and the Meckel cave. It may also cause protrusion of cisternal structures of the Meckel cave through the skull base foramina, which could result in indentation or a bilobed appearance of the Meckel cave. We investigated the changes in the Meckel cave in patients with proved idiopathic intracranial hypertension versus healthy controls.
\end{abstract}

MATERIALS AND METHODS: We studied 75 patients with a diagnosis of idiopathic intracranial hypertension and 75 age-and sexmatched healthy controls. The transverse diameter of Meckel cave was measured in the axial and coronal planes of T2-weighted MR imaging sequences, and comparison was made between the 2 groups.

RESULTS: The mean diameters of the Meckel cave on the coronal T2 plane in patients with idiopathic intracranial hypertension were $5.21 \pm 1.22 \mathrm{~mm}$ on the right side and $5.16 \pm 0.90 \mathrm{~mm}$ on the left side, while in the control group, they measured $3.89 \pm$ $0.62 \mathrm{~mm}$ and $4.09 \pm 0.68 \mathrm{~mm}$, respectively $(P$ value $<.001)$. Of 75 patients with an approved diagnosis of idiopathic intracranial hypertension, $57(76 \%)$ showed an indented Meckel cave as opposed to $21(28 \%)$ in the control group.

CONCLUSIONS: Our results confirm for the first time that the shape and size of the Meckel cave can be used as sensitive and specific diagnostic imaging markers for the diagnosis of idiopathic intracranial hypertension.

ABBREVIATIONS: AP = anterior-posterior; AUC = area under the receiver operating characteristic curve; $I \mathrm{H}=$ idiopathic intracranial hypertension; $\mathrm{MC}=$ Meckel cave; $\mathrm{PE}=$ papilledema; $\mathrm{TSS}=$ transverse sinus stenosis

diopathic intracranial hypertension (IIH), also known as pseudotumor cerebri, is a syndrome with signs and symptoms of increased intracranial pressure. However, a primary cause such as space-occupying brain lesions, including brain parenchymal mass or enlarged ventricles, has not been identified. The etiology and diagnostic criteria of IIH have not been clearly described. The incidence of IIH is reported to be increasing, starting from 0.03 to 2.36 per 100,000 per year. ${ }^{1,2}$ A few clinical diagnostic criteria of IIH have been proposed. ${ }^{3-5}$ Some of the clinical criteria

Received October 14, 2019; accepted after revision May 19, 2020.

From the Department of Diagnostic Radiology (A.K., A.A., O.A., P.R., X.Z., R.E.G., R.F.R.), University of Texas at Houston, Houston, Texas; University of Texas Medical School Health Science Center Houston (K.C.S., S.J.C.), Houston, Texas; Neuroimaging Laboratory at Mallinckrodt Institute of Radiology (F.R.), Washington University School of Medicine, St. Louis, Missouri; and Rice University (A.G.), Houston, Texas.

Please address correspondence to Arash Kamali, MD, Department of Diagnostic Radiology, Neuroradiology Section, University of Texas at Houston, 6431 Fannin St, Houston, TX 77030; e-mail: arash.kamali@uth.tmc.edu

http://dx.doi.org/10.3174/ajnr.A6682 for the diagnosis of $\mathrm{IIH}$ are described as unilateral or bilateral papilledema (PE) with or without sixth nerve palsy, normal neurologic examination and CSF analysis findings, the absence of brain parenchymal pathology or enlarged ventricles, and elevated CSF opening pressure. ${ }^{4}$ Neuroimaging is critical to exclude secondary causes of intracranial hypertension, including brain pathologies such as brain mass, hemorrhage, hydrocephalus, and so forth. Brain imaging is also helpful to identify imaging findings of elevated intracranial pressure. Some of the imaging findings of elevated intracranial pressure that support the diagnosis of $\mathrm{IIH}$ when no secondary cause is identified include the following: posterior orbital globe flattening, optic nerve head protrusion into the back of orbital globe (PE), dilated optic nerve sheaths, empty/ partial empty sella, bilateral transverse sinus stenosis (TSS), cerebellar tonsillar herniation, meningoceles/encephaloceles, and spontaneous CSF leakage. IIH may be diagnosed in the presence of PE or sixth nerve palsy if the clinical criteria for IIH and elevated opening CSF pressure are satisfied. In the absence of PE 


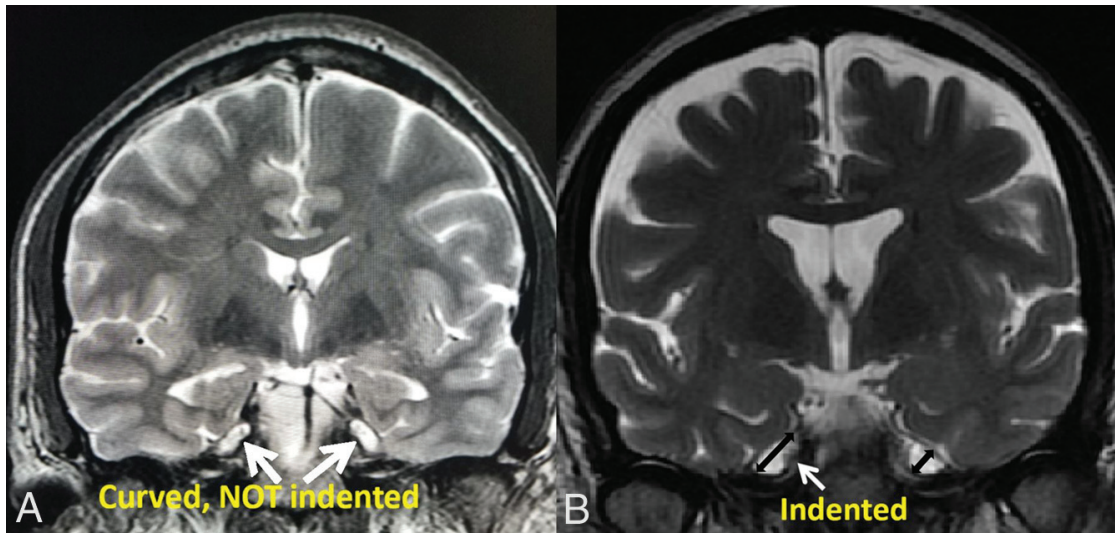

FIG 1. Two coronal T2-weighted images of the Meckel cave in a healthy subject $(A)$ versus a patient with $\mathrm{IIH}(B)$. The white arrows in $A$ represent the curvature of the Meckel cave and no indentation in a healthy subject. The white arrow in $B$ demonstrates an acute angle of indentation and a bilobed appearance of the Meckel cave in a patient with $\mathrm{IIH}$. The 2-way arrows in B demonstrate the craniocaudal diameter (along the oblique axis of the right $\mathrm{MC}$ ) and transverse diameter of the left MC (perpendicular to both walls in the widest segment of the MC on the coronal plane). and dilation of the optic nerve sheaths. Elevated intracranial pressure also pushes the structures into the skull base foramina and results in encephalocele/ meningocele. ${ }^{8}$ Protrusion of some structures such as the Meckel cave or cerebellar tonsils may also be seen through the skull base foramina. This may result in an indented or bilobed appearance of the Meckel cave. The purpose of this study was to investigate whether the shape (bilobed or indented appearance of the Meckel cave) or the size/volume of the Meckel cave on T2-weighted MR imaging sequences could serve as a noninvasive diagnostic imaging marker for the diagnosis of IIH.

\section{MATERIALS AND METHODS}

Institutional review board approval was

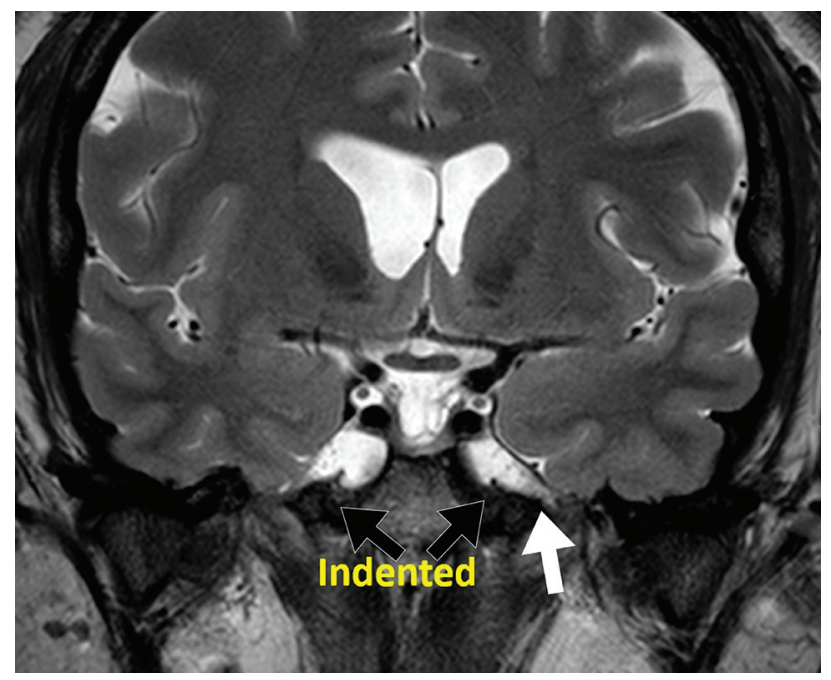

FIG 2. Coronal $T 2$ view of a dilated $M C$ in a patient with $I I H$. The black arrows represent the indentation of the Meckel cave. The white arrow shows the dilated cisternal space of the V3 segment of the left trigeminal nerve and points to the foramen ovale where the V3 nerve exits the intracranial space at the skull base. Dilation of this cisternal space may contribute to the indented appearance of the MC in patients with IIH.

or sixth nerve palsy, IIH is considered "probable" if at least 3 neuroimaging criteria are present, given that other clinical criteria and raised opening CSF pressure are satisfied. ${ }^{4,6}$

Overall, no single imaging finding has high specificity and sensitivity enough to be considered diagnostic for IIH. Each imaging finding on its own lacks either sensitivity or specificity. For instance, the posterior scleral flattening has $100 \%$ specificity but only $66 \%$ sensitivity for a diagnosis of $\mathrm{IIH}^{7}$ However, a combination of imaging criteria on MR imaging will increase the sensitivity for a diagnosis of IIH. Increased intracranial pressure forces CSF into the cisternal spaces and results in dilation of the perineural cisternal spaces such as the optic nerve sheath and the Meckel cave (MC). This results in enlargement of the Meckel cave obtained. Seventy-five adult patients (6 male and 69 female patients between 18 and 59 years of age) with a confirmed diagnosis of intracranial hypertension and documented elevated lumbar puncture opening CSF pressure $\left(\geq 250 \mathrm{~mm}\right.$ of $\mathrm{H}_{2} \mathrm{O}$ ) were selected retrospectively for this study. The subjects were retrospectively selected at the Memorial Hermann Hospital, Texas Medical Center, Houston from 2015 to 2018. Subjects with any intracranial abnormality on MR imaging such as evidence of mass/lesion, demyelination, infection, vascular malformation, or prior trauma were excluded from the study. Subjects who were clinically diagnosed with brain infection such as meningitis or encephalitis were also excluded. A second group of 75 age-matched (18-59 years of age) healthy adults who presented to the emergency department with headache with unremarkable clinical and imaging examination findings were also selected as the healthy control group retrospectively. The opening CSF pressure values were only available for about half of the control group (32 subjects) who had documented normal opening pressure. However, subjects with chronic headaches and multiple brain scans were excluded from the control group to eliminate the possibility of elevated intracranial pressure. We also excluded subjects with visual changes, dizziness, and tinnitus reported, along with headache from the healthy control group. We looked for indentation of the Meckel cave on the coronal T2-weighted sequences in both groups (Fig $1 B$ and 2). The indentation was considered positive if there was an acute angle or bilobed appearance of the Meckel cave on the coronal T2 sequences (Fig $1 B$ and 2). The smooth curvature of the Meckel cave was considered negative for indentation (Fig 1A).

The transverse, anterior-posterior (AP), and craniocaudal diameters of the Meckel cave on both sides were measured by wall-to-wall measurements on the axial and coronal T2 planes (Figs 1 and 3). Given the cuboid appearance of the MC structure, we estimated the volume of the MC by multiplying the AP, transverse, and craniocaudal values. The measurements were performed by a neuroradiologist and a medical student with high 


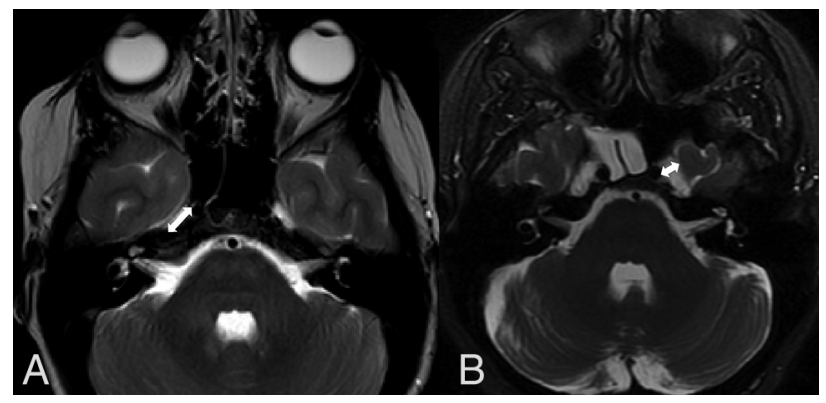

FIG 3. The appearance of the Meckel cave in a healthy person $(A)$ versus a patient with $\mathrm{IIH}(B)$ on the axial $\mathrm{T} 2$ planes. The 2-way arrows represent the wall-to-wall measurement of the Meckel cave in the $\operatorname{AP}(A)$ and transverse $(B)$ diameters.
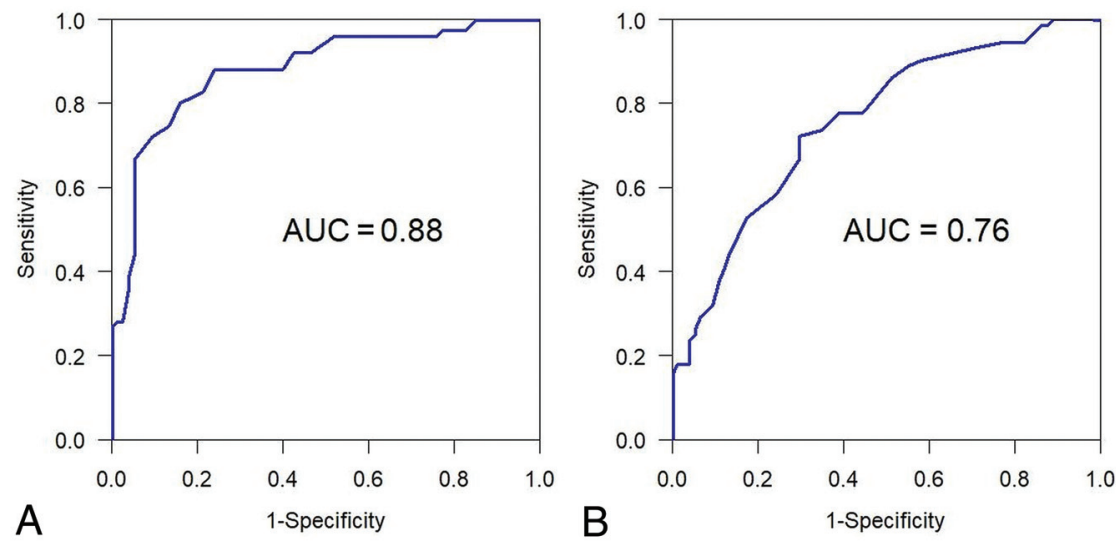

FIG 4. Figs 4-7. The AUC was used to evaluate the overall differentiability of a marker to identify those with IIH from healthy controls. The optimal cutoff point for the marker was obtained by evaluating the Youden index. On the basis of the optimal cutoff point, we reported estimates of sensitivity, specificity, positive predictive value, and negative predictive value, together with their $95 \%$ Wald confidence intervals. Maximum transverse values. $A$, coronal data. $B$, axial data.
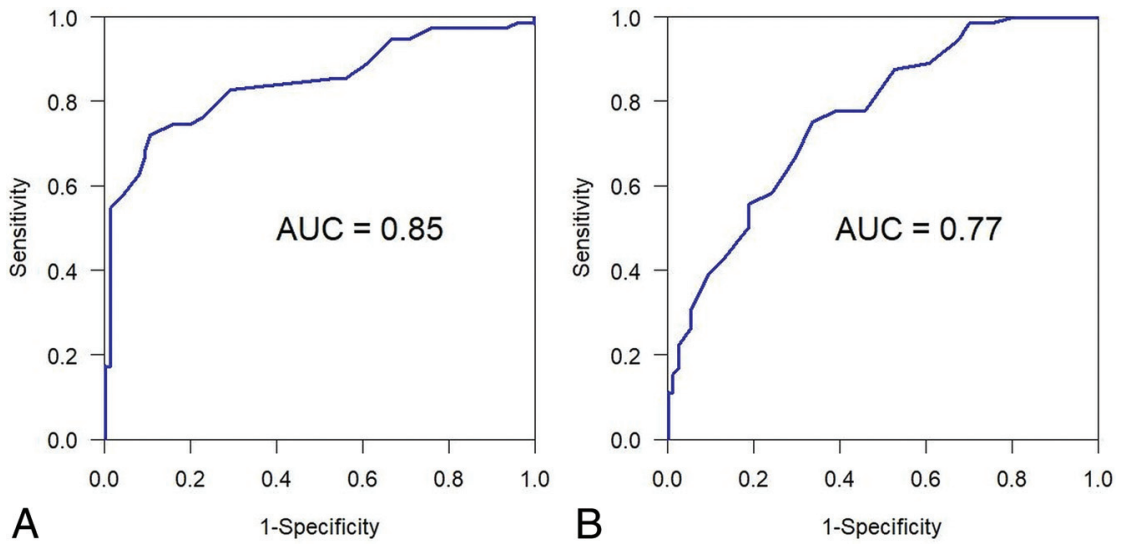

FIG 5. Right transverse values. A, Coronal data. $B$, Axial data.

reproducibility results. Both measurements were performed by two experienced neuroradiologists blinded to both groups for the revisions. The results were analyzed by our in-house statistician.

The area under the receiver operating characteristic curve (AUC) was used to evaluate the overall differentiability of a marker to distinguish those with IIH from healthy controls (Figs 4-7). The optimal cutoff point for the marker was obtained by evaluating the Youden index. On the basis of the optimal cutoff point, we reported estimates of sensitivity, specificity, positive predictive value, and negative predictive value, together with their 95\% Wald confidence intervals. We also looked for other imaging signs of IIH (Table 1) such as an empty/partial empty sella (loss of height of the pituitary gland below one-third height of the sella turcica), optic nerve sheath dilation (optic nerve sheath diameter $\geq 6 \mathrm{~mm}$ ), posterior globe flattening, papilledema (protrusion of the optic nerve head into the posterior globe), and bilateral TSS.

\section{RESULTS}

A total of 150 subjects were observed, with 75 subjects in the IIH group and 75 patients in the control group (Table 2). This table shows that the IIH and healthy control groups are age-matched; however, there is a sex mismatch between the 2 groups. Of 75 patients with diagnoses of IIH, 57 (76\%) had an indented MC and 18 (24\%) were negative for indentation. In the healthy control group, of 75 subjects, 21 (28\%) had indentation and 54 subjects $(72 \%)$ were negative for indentation (Table 3). A positive indentation of the MC showed $76 \%$ sensitivity and $72 \%$ specificity for a diagnosis of IIH. The positive predictive value of an indented MC for the diagnosis of $\mathrm{IIH}$ was $73 \%$, and the negative predictive value was $75 \%$.

In patients with diagnoses of $\mathrm{IIH}$, the maximal transverse, AP, and craniocaudal diameters along with volume measurement of the bilateral Meckel cave were recorded and compared with those of healthy controls on the axial and coronal T2-weighted MR imaging planes. The axial T2approach acquired data showed a maximum Meckel cave transverse diameter of $5.49 \pm 1.11 \mathrm{~mm}$ in patients with $\mathrm{IIH}$ and a transverse diameter of $4.14 \pm 0.72 \mathrm{~mm}$ in the control group with a $P$ value $<.001$. In the right Meckel cave, patients with IIH had a mean diameter of $5.14 \pm 1.21 \mathrm{~mm}$, and controls had a mean diameter of $3.94 \pm 0.73 \mathrm{~mm}$ with a $P$ value $<.001$. The left Meckel cave mean measurement of the patients with $\mathrm{IIH}$ was $4.94 \pm 0.96 \mathrm{~mm}$, and controls measured $3.88 \pm 0.75 \mathrm{~mm}$ with a $P$ value $<.001$. Our axial T2 results showed a cutoff value of $4.3 \mathrm{~mm}$ in the right Meckel cave with $75.0 \%$ sensitivity and $66 \%$ specificity. A cutoff of $4.2 \mathrm{~mm}$ can be used on the left Meckel cave with $79.0 \%$ sensitivity and $60 \%$ specificity. Summary 
statistics for measurement values of the MC in patients with IIH and controls are provided in Table 4.

The acquired measurement data on the coronal T2 plane showed a maximum Meckel cave transverse diameter of $5.62 \pm 1.03 \mathrm{~mm}$ in patients with $\mathrm{IIH}$, and a transverse diameter of $4.22 \pm 0.69 \mathrm{~mm}$ in the control group, with a $P$ value $<.001$. The right Meckel cave in patients with IIH had a mean diameter of $5.21 \pm 1.22 \mathrm{~mm}$, and in controls, it had a mean diameter of $3.99 \pm 0.62 \mathrm{~mm}(P$ value $<.001)$. The mean measurement of the left Meckel cave in patients with $\mathrm{IIH}$ was $5.16 \pm 0.90 \mathrm{~mm}$, and in controls, it measured $4.09 \pm 0 . \mathrm{mm}(P$ value $<.001)$. Our coronal T2 results showed a cutoff value of $4.60 \mathrm{~mm}$ in the right Meckel cave with $80.0 \%$ sensitivity and $89.3 \%$ specificity. A cutoff of $4.80 \mathrm{~mm}$ can be used on the left Meckel cave with $68.0 \%$ sensitivity and $85.3 \%$ specificity.

The right Meckel cave in patients with IIH had a mean height of $11.51 \pm 1.78 \mathrm{~mm}$, and in controls, it had a mean diameter of $9.51 \pm 1.46 \mathrm{~mm}(P$ value $<.001)$ on the coronal T2 sequences. The mean measurement of the left Meckel cave height in patients with $\mathrm{IIH}$ was $11.93 \pm 1.9 \mathrm{~mm}$, and in the controls, it measured $9.53 \pm 1.43 \mathrm{~mm}(P$ value $<.001)$.

In patients with $\mathrm{IIH}$, the mean $\mathrm{AP}$ diameter of the right $\mathrm{MC}$ on the axial T2 sequences was $12.37 \pm 2.43 \mathrm{~mm}$, and in controls, it measured $9.68 \pm 1.43 \mathrm{~mm}(P$ value $<.001)$. The mean AP diameter of the left MC measured $12.92 \pm 2.70 \mathrm{~mm}$ in the $\mathrm{IIH}$ group versus $9.55 \pm 1.47 \mathrm{~mm}$ in controls $(P$ value $<.001)$. In patients with $\mathrm{IIH}$, the mean volume of the right $\mathrm{MC}$ measured $747.3 \pm 293 \mathrm{~mm}^{3}$ versus $368.7 \pm 121 \mathrm{~mm}^{3}$ in the control group $(P$ value $<.001)$. The mean volume of the left MC measured $775.4 \pm 278 \mathrm{~mm}^{3}$ in IIH group versus $357.2 \pm 115 \mathrm{~mm}^{3}$ in the control group $(P$ value $<.001)$. The maximum value was also selected as the larger value between the right and left. The maximum value for the volume in the IIH group measured $938.4 \pm 333 \mathrm{~mm}^{3}$ versus $424.0 \pm 131 \mathrm{~mm}^{3}$ in healthy controls $(P$ value $<.001)($ Table 4$)$

The volume markers had significantly higher AUC values than the AP, transverse, or craniocaudal diameters (Table 5). Among volume markers (right, left, maximum), maximum volume had a significantly higher AUC than the right or left MC volumes (Table 5).

We also estimated the Spearman correlation coefficient to see if the CSF opening pressure correlated with the size of the MC. The Spearman correlation coefficient was only -0.10 for the right $\mathrm{MC}$ volume and CSF pressure, and -0.05 for the left MC volume and CSF opening pressure (Table 6). Correlation coefficients were very close to zero. We may conclude that opening CSF pressure is not correlated with right or left MC volumes.

We also checked to see whether there was any correlation between aging and the size of the MC. Our results showed that the Spearman correlation coefficient was only 0.06 $(P$ value $=.58)$ for the MC volume and aging in the healthy control group and 0.007 ( $P$ value $=.96)$ in the IIH group. The sample correlation coefficients are very close to zero, and we may conclude that aging is not correlated with the volume of the $\mathrm{MC}$ in either group. We further looked for a possible effect of sex on the size of the MC in the control group. No significant difference was found between the volume of the $\mathrm{MC}$ in 15 male controls compared with the 60 female controls $(P$ value $=$ 0.96). This verifies that mismatched

FIG 6. Left transverse values. $A$, Coronal data. $B$, Axial data.
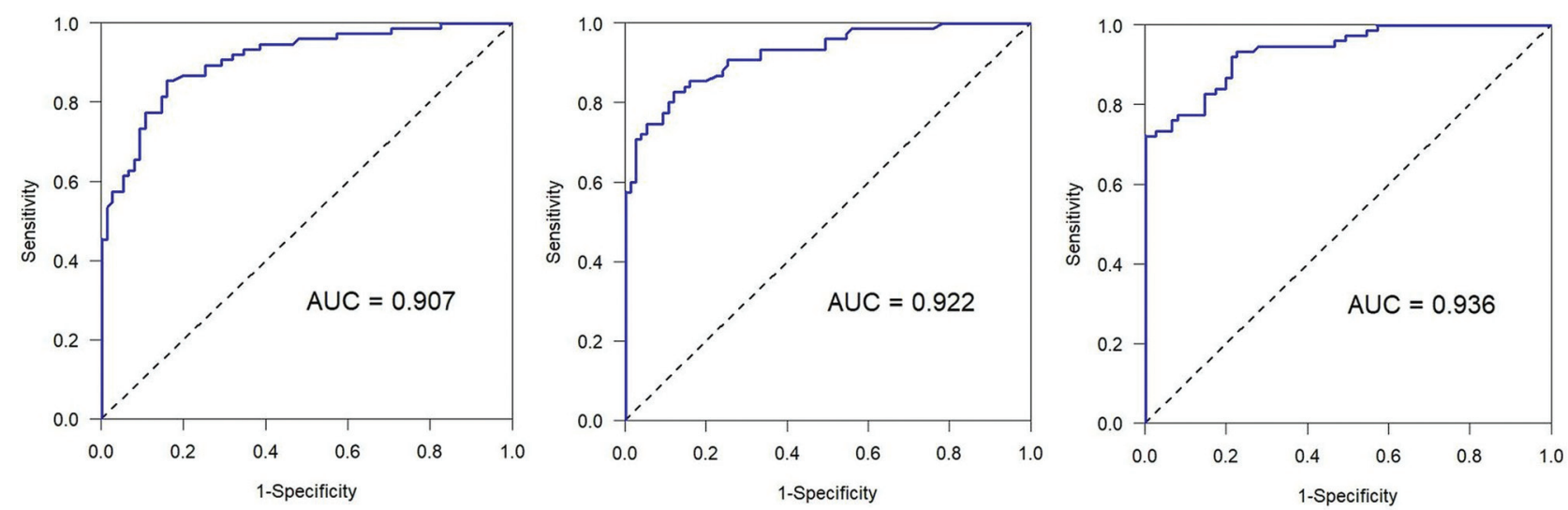

FIG 7. Receiver operating characteristic curves for MC volumes: right MC (right), left MC (center), and maximum values (left). 
Table 1: Incidence, sensitivity, and specificity of imaging findings in IIH and control groups

\begin{tabular}{lcccc}
\hline \multicolumn{1}{c}{ Imaging Signs } & IIH & Controls & Sensitivity & Specificity \\
\hline Optic nerve sheath dilation & $63(84 \%)$ & $12(16 \%)$ & 84 & 84 \\
Empty/partial empty sella & $69(92 \%)$ & $20(26 \%)$ & 92 & 74 \\
Posterior scleral flattening & $41(55 \%)$ & 0 & 55 & 100 \\
Papilledema & $34(45 \%)$ & 0 & 45 & 100 \\
Bilateral TSS & $55(73 \%)$ & $6(8 \%)$ & 73 & 92 \\
Enlarged MC & $56(75 \%)$ & $11(14 \%)$ & 75 & 86 \\
\hline
\end{tabular}

Table 2: Demographics for 2 groups

\begin{tabular}{lccc}
\hline & IIH $(\boldsymbol{n}=75)$ & Healthy Controls $(\boldsymbol{n}=75)$ & $\boldsymbol{P}$ Value \\
\hline Age (mean $\pm \mathrm{SD})(\mathrm{yr})$ & $33.84 \pm 9.15$ & $34.65 \pm 9.37$ & .59 \\
Male sex & $6(8 \%)$ & $15(20 \%)$ & .034 \\
LP opening pressure (median) (IQR) & $29(24-38)$ & Not Available & \\
\hline
\end{tabular}

Note:-IIH indicates idiopathic intracranial hypertension patients; LP, lumbar puncture; IQR, interquartile range.

Table 3: Meckel cave indentation ${ }^{\mathrm{a}}$

\begin{tabular}{lccc}
\hline & Yes & No & Total \\
\hline $\mathrm{IIH}$ & 57 & 18 & 75 \\
Healthy controls & 21 & 54 & 75 \\
\hline
\end{tabular}

${ }^{a}$ Seventy-six percent sensitivity, $72 \%$ specificity, positive predictive value of $73 \%$ and negative predictive value of $75 \%$ for the diagnosis of $\mathrm{IH}$.

Table 4: Descriptive statistics in IIH patients $(n=75)$ and controls $(n=75)^{a}$

\begin{tabular}{lccc}
\hline \multicolumn{1}{c}{ Variable } & Patients with IIH & Controls & $P$ Value \\
\hline Right MC height & $11.51 \pm 1.78$ & $9.51 \pm 1.46$ & $<.001$ \\
Left MC height & $11.93 \pm 1.90$ & $9.53 \pm 1.43$ & $<.001$ \\
Max MC height & $12.50 \pm 1.92$ & $9.97 \pm 1.51$ & $<.001$ \\
Right MC AP & $12.37 \pm 2.43$ & $9.68 \pm 1.43$ & $<.001$ \\
Left MC AP & $12.92 \pm 2.70$ & $9.55 \pm 1.47$ & $<.001$ \\
Max MC AP & $13.41 \pm 2.48$ & $10.13 \pm 1.43$ & $<.001$ \\
Right MC transverse & $5.14 \pm 1.21$ & $3.94 \pm 0.73$ & $<.001$ \\
Left MC transverse & $4.94 \pm 0.96$ & $3.88 \pm 0.75$ & $<.001$ \\
Max MC transverse & $5.49 \pm 1.11$ & $4.14 \pm 0.72$ & $<.001$ \\
Right volume & $747.3 \pm 293$ & $368.7 \pm 121$ & $<.001$ \\
Left volume & $775.4 \pm 278$ & $357.2 \pm 115$ & $<.001$ \\
Max volume & $938.4 \pm 333$ & $424.0 \pm 131$ & $<.001$ \\
\hline
\end{tabular}

Note:-Max indicates maximum.

${ }^{a}$ Mean \pm SD are shown. Summary statistics for anterior-posterior (AP) and transverse diameters of $M C$ on axial T2, and craniocaudal diameter of $M C$ on coronal T2 weighted sequences. Max is larger value between right and left. Volume is product of height, AP, and transverse diameters.

Table 5: AUC estimates for differentiating patients with IIH from controls

\begin{tabular}{lll}
\hline \multicolumn{1}{c}{ Variable } & AUC & \multicolumn{1}{c}{$95 \% \mathrm{Cl}$} \\
\hline Right MC height & 0.816 & $(0.748-0.884)$ \\
Left MC height & 0.852 & $(0.793-0.911)$ \\
Max MC height & 0.850 & $(0.789-0.910)$ \\
Right MC AP & 0.830 & $(0.768-0.893)$ \\
Left MC AP & 0.861 & $(0.804-0.917)$ \\
Max MC AP & 0.874 & $(0.821-0.927)$ \\
Right MC transverse & 0.810 & $(0.740-0.879)$ \\
Left MC transverse & 0.807 & $(0.737-0.877)$ \\
Max MC transverse & 0.852 & $(0.791-0.913)$ \\
Right volume & 0.907 & $(0.861-0.954)$ \\
Left volume & 0.922 & $(0.881-0.964)$ \\
Max volume & 0.936 & $(0.900-0.972)$ \\
\hline
\end{tabular}

Note:-Max indicates maximum. sex between the IIH and healthy groups does not affect the results.

Our results demonstrated that the empty/partial empty sella has the highest sensitivity (92\%) for the detection of IIH followed by optic nerve sheath dilation (84\%), an enlarged MC (75\%), and the TSS (73\%). Posterior scleral flattening and PE have the highest specificity for the diagnosis of IIH (100\%), followed by TSS (92\%), enlargement of the MC (86\%), optic nerve sheath dilation (84\%), and empty/partial empty sella (74\%) (Table 1).

\section{DISCUSSION}

Aaron et $\mathrm{al}^{9}$ evaluated patients with spontaneous CSF leak and elevated intracranial pressure and measured the AP and transverse diameters of the MC only on the axial T2-weighted sequences. They concluded that the size of the MC was larger in patients with elevated intracranial pressure compared with healthy controls. The current results confirm the findings of Aaron et al, ${ }^{9}$ in 2017, showing that the size of MC is increased in the IIH group compared with healthy controls. However, our results contradict the results of Degnan et al, ${ }^{10}$ who reported a decrease in size of the MC in patients with IIH compared with healthy controls. To the best of our knowledge, our study is the first to evaluate the volumes of the Meckel cave (using 3D measurements) and to introduce the shape of the MC (indentation or bilobed appearance) as a diagnostic imaging marker in patients with IIH versus a healthy population. The current study confirms that there is a significant difference in the shape and size of the Meckel cave in patients with IIH versus the healthy control group, which may be used as both sensitive and specific noninvasive imaging markers in the diagnosis of IIH.

Our results showed statistically significant differences in the size of both the right and left Meckel cave between the diseased group and healthy controls, using measurements on both the axial and coronal T2-weighted MR imaging sequences. A novel finding of the current study was the higher sensitivity and specificity of the coronal $\mathrm{T} 2$ measurements compared with the axial T2 measurement approach $(80.0 \%$ sensitivity and $89.3 \%$ specificity on coronal measurement versus $75.0 \%$ sensitivity and $66 \%$ specificity on the axial measurement). We believe this discrepancy is related to the anatomic orientation of the MC structure from the posterior-lateral to the anterior-medial. Because the MC is a longer structure in the anterior-posterior dimension than the craniocaudal dimension, the coronal $\mathrm{T} 2$ sequences have a better chance of cutting through the MC than the axial T2 sequences, given that the section thicknesses would be the same.

The values of the AP diameter of the MC in the study by Aaron et $\mathrm{al}^{9}(14.7 \pm 2.8 \mathrm{~mm}$ in $\mathrm{IIH}$ and $11.5 \pm 1.9 \mathrm{~mm}$ in the control group) were comparable with our results of AP diameters $(13.41 \pm 2.48 \mathrm{~mm}$ in the $\mathrm{IIH}$ group and $10.13 \pm 1.4 \mathrm{~mm}$ in the control group). However, the values for the transverse diameter of the MC by Aaron et al $(6.3 \pm 1.8 \mathrm{~mm}$ in the IIH group and $5.1 \pm 0.9 \mathrm{~mm}$ in the healthy control group) were larger than our 
Table 6: Estimated Spearman correlation coefficients $(n=75)^{\mathrm{a}}$

\begin{tabular}{lcccc}
\hline \multicolumn{1}{c}{ Variable 1 } & Variable 2 & $\begin{array}{c}\text { Estimated Spearman } \\
\text { Correlation Coefficient }\end{array}$ & 95\% Cl & $\begin{array}{c}\boldsymbol{P} \\
\text { Value }\end{array}$ \\
\hline Right MC volume & CSF opening pressure & -0.104 & $-0.323-0.127$ & .38 \\
Left MC volume & CSF opening pressure & -0.050 & $-0.274-0.179$ & .67 \\
\hline
\end{tabular}

${ }^{a}$ The Spearman correlation coefficient was only -0.10 for right MC volume and CSF pressure, and -0.05 for left MC volume and CSF opening pressure. Correlation coefficients are very close to zero. We may conclude that opening CSF pressure is not correlated with right or left MC volumes.

right versus $4.09 \pm 0.68 \mathrm{~mm}$ on the left in the coronal $\mathrm{T} 2$ approach and $4.01 \pm 0.72 \mathrm{~mm}$ on the right and $4.03 \pm 0.82 \mathrm{~mm}$ on the left on the axial T2 measurement approach). However, in the IIH group, the Meckel cave had a comparable size in both coronal and axial T2 measurement approaches. In our experience, the MC was markedly diminutive or

results by about $20 \%$ (about $1 \mathrm{~mm})(5.17 \pm 0.99 \mathrm{~mm}$ in the $\mathrm{IIH}$ group and $3.94 \pm 0.73 \mathrm{~mm}$ in healthy controls). This difference may be related to multiple factors. The study by Aaron et al primarily measured the left MC structures which, in our study, are generally larger than the right MC structures, specifically in healthy controls. Aaron et al also excluded the subjects in whom the axial T2 sequences were not optimal for measurement. Given that the study by Aaron et al did not specify the section thickness of their imaging protocol, this issue might have resulted in a selection bias by selecting only subjects with larger MCs, which would be easier to measure on the axial plane. In our institution, the axial and coronal T2 sequences have the same section thicknesses of a 3- to 5-mm range. Our results also showed that the mean transverse diameter of the $\mathrm{MC}$ is larger on the coronal measurements compared with the axial approach (5.62 \pm $1.03 \mathrm{~mm}$ on the coronal and $5.17 \pm 0.99 \mathrm{~mm}$ on the axial plane). Given the oblique orientation of the MC structures anatomically (Fig 1), the discrepancy is likely related to obliquity (not perpendicular to the axis) of the wall-to-wall measurement of the transverse diameter of the MC on the coronal approach. On the axial measurement, however, the wall-to-wall measurement is the shortest because the measurement is perpendicular to the axis of the MC (Fig 3).

Our results showed a significant difference in the volume of the MC between the 2 groups (healthy versus IIH) (mean value of volume $=938.4 \pm 332.8 \mathrm{~mm}^{3}$ in the IIH group versus $424.0 \pm$ $131.5 \mathrm{~mm}^{3}$ in controls with a $P$ value $\left.<.001\right)$. The volume markers had significantly higher AUC values than the AP, transverse, or craniocaudal diameters (Table 5), which is a novel finding in this study compared with prior ones. However, measuring the transverse diameter of the MC on the coronal T2 sequences would be much more feasible for the reading radiologist than estimating the volume, given the high differentiability of both markers (AUC value $=0.93$ for volume versus 0.85 for the transverse diameter). Given that the MC structure is slightly longer in the AP than in the craniocaudal diameter, measuring the AP diameter of the MC on axial T2-weighted imaging is less feasible than measuring the transverse or craniocaudal diameters on the coronal T2 planes. No correlation was found between the size of the MC with aging or CSF opening pressure in either IIH or healthy control groups; this is an additional novel finding of the current study.

Another novel finding of the current study is the developmental asymmetry in the diameter of the Meckel cave in healthy controls. Our results showed that the left Meckel cave was slightly larger than the right in the control group in both approaches on the axial and coronal T2 measurements $(3.89 \pm 0.62 \mathrm{~mm}$ on the hypoplastic in a few subjects with diagnoses of IIH and in healthy controls. This finding likely represents a normal developmental variable. Given the diminutive size or hypoplasia of the MC in these subjects, higher intracranial CSF pressure leaves no space to dissect at the skull base as opposed to others with well-developed MC cisternal structures. Our results showed a bilobed and indented appearance of the MC with a notch in the middle of the cistern in $76 \%$ ( 57 of 75 ) of patients with IIH as opposed to $28 \%$ (21 of 75) of the healthy controls (76\% sensitivity and $72 \%$ specificity in the diagnosis of IIH) (Table 3).

The bilobed appearance of the MC is believed to be related to the increased intracranial pressure resulting in pushing the cisternal structure of the MC into the osseous opening of the skull base. This mechanism is believed to be the same one causing an increased incidence of meningocele/encephalocele in patients with IIH. ${ }^{8}$ Our results revealed that a quick observation of the $\mathrm{MC}$ on the coronal MR imaging sequences and identifying the indentation (or bilobed appearance) of the MC yielded a 73\% (positive predictive value) chance of an accurate diagnosis of IIH. The absence of a bilobed or indented appearance of the MC on coronal sequences can rule out the diagnosis of IIH by $75 \%$ probability (negative predictive value). Because the cisternal space surrounding the mandibular branch of the trigeminal nerve is the anatomic extension of the Meckel cave, the indented appearance of the MC may be, in part, due to extension of CSF from the MC into the proximal cisternal space of the mandibular branch of the trigeminal nerve secondary to increased CSF pressure (Fig 2).

The role of neuroimaging is to rule out the secondary causes of intracranial hypertension, including space-occupying lesions such as hemorrhage, tumor, or vascular anomalies (dural arteriovenous fistula, arteriovenous malformation). Non-space-occupying pathologies such as infection or inflammation may also result in secondary elevation of intracranial pressure. Our results showed that posterior scleral flattening and PE are the most specific imaging findings ( $100 \%$ specificity), followed by bilateral TSS (92\% specificity), an enlarged MC (86\%), optic nerve sheath dilation (84\%), and an empty/partial empty sella (75\%). Despite the lowest specificity (75\%), an empty/partial empty sella showed the highest sensitivity among the imaging findings (92\% sensitivity) followed by optic nerve sheath dilation (84\%), enlarged MC (75\%), and TSS (73\%). Posterior scleral flattening and PE showed the lowest sensitivity ( $54 \%$ and $45 \%$, respectively).

Our study revealed that enlargement of the Meckel cave has about $75 \%$ sensitivity and about $86 \%$ specificity for diagnosis of $\mathrm{IIH}$, which has the third highest sensitivity and specificity among the preceding imaging findings. Bilateral TSS was reported to be the most common and sensitive imaging finding of IIH among 
all imaging findings (in $94 \%$ of patients with IIH compared with $3 \%$ of controls). ${ }^{11}$ However, our study showed that despite the high specificity (92\%), TSS has only $73 \%$ sensitivity in the diagnosis of IIH. An empty sella/partial empty sella is reported as a relatively sensitive imaging finding in patients with $\mathrm{IIH}$, especially in younger adults (about 80\% sensitivity). However, the definition of a partial empty sella, which is a more sensitive and less specific finding than a complete empty sella, is variable among different studies with a wide range of reported specificity (44\%-80\% sensitivity and $<70 \%$ specificity). Despite the lowest specificity (75\%), the empty/partial empty sella showed the highest sensitivity among the imaging findings ( $92 \%$ sensitivity) in our study. This finding is not specific to IIH and is commonly seen in the general population, specifically in older adults due to age-related atrophy.

Orbital findings are also common in this population. Flattening of the posterior aspect of the globe or scleral flattening has a sensitivity of $66 \%$, and PE has even less sensitivity, about $43 \%$. ${ }^{6,12}$ Among orbital findings, posterior scleral flattening and $\mathrm{PE}$ have the highest specificity for the diagnosis of $\mathrm{IIH}$, which is close to $100 \% .{ }^{12}$ However, posterior scleral flattening and PE both have poor sensitivity. ${ }^{12}$ None of the imaging markers exhibited a combination of both high sensitivity and specificity in the diagnosis of $\mathrm{IIH}$. However, a combination of the above imaging findings would offer highly sensitive and specific approaches for the diagnosis of $\mathrm{IIH}$, superior to any single imaging marker. This may help radiologists suggest a diagnosis of IIH to prompt an early diagnosis and prevent permanent damage caused by this disease. The combination of imaging findings may also help reduce overdiagnosis of $\mathrm{IIH}$ by radiologists, which might prevent patients from undergoing unnecessary invasive procedures such as lumbar puncture.

To date, the etiology of IIH is unknown, and the exact mechanism is not well-understood. Some of the risk factors for IIH include female sex, obesity, and hypervitaminosis A. Some of the proposed pathophysiologies for IIH are increased secretion of CSF, disordered CSF absorption, or increased intracranial venous pressure. Other etiologies include venous sinus stenosis/thrombosis or venous sinus compression leading to blockage of CSF distribution. Normal CSF flow is known to be secreted by the choroid plexus, travel through the subarachnoid space, and then exit through arachnoid granulations or glymphatic pathway. ${ }^{13}$ The glymphatic pathway (also known as the glial-lymphatic pathway) acts as a pseudolymphatic system in the brain. ${ }^{14}$ The CSF flows along the perivascular spaces and then into the interstitium via aquaporin $4 .^{14,15}$ Glymphatic dysfunction is believed to be due to aquaporin 4 dysfunction, leading to decreased distribution of CSF in brain parenchyma. ${ }^{14,15}$ The most common presenting symptom in patients with a diagnosis of IIH is headache, which worsens when lying down or bending over. ${ }^{16}$ Other features include visual disturbances, such as blurred vision, photophobia, diplopia, transient visual fluctuations, or visual loss due to ischemic optic neuropathy. ${ }^{17}$ Pulsatile tinnitus, dizziness, neck pain, seizure, paresthesia, weakness, or cognitive impairment are also nonspecific symptoms associated with IIH.

Most patients presenting with any of the specified symptoms will end up undergoing brain imaging such as brain CT and/or MR imaging. Radiologists and specifically neuroradiologists should be sensitive to imaging manifestations of IIH in brain MR imaging to prompt an early diagnosis and treatment to avoid complications such as chronic headache and vision loss.

One of the limitations of the current study is variable section thicknesses of the T2 sequences in our institution (3$5 \mathrm{~mm}$ ). However, the axial and coronal T2 planes are of identical section thicknesses on a single scan in our institution. Larger section thickness may obscure the visibility of the MC, compromising the visibility of indentation of the $\mathrm{MC}$ in 1 plane. Because the anterior-posterior length of the MC measures about $12-15 \mathrm{~mm}$, even with the section thickness of $5 \mathrm{~mm}$, at least 2 coronal planes will be acquired through the MC structures. In addition, when the indentation of the MC was not appreciated in the coronal plane, we checked the axial T2 plane to avoid a false-negative result, given that the bilobed appearance of the MC is visible on both planes most of the time.

The opening CSF pressure value was not available for about half of the healthy control subjects included in the current study to rule out elevated CSF pressure. However, we excluded subjects with chronic headaches and multiple brain scans to eliminate the possibility of elevated intracranial pressure. We also excluded subjects with visual changes, dizziness, and tinnitus reported along with headache for the healthy controls. In the absence of a significant difference in the size of the MC between subjects in the control group with and without documented normal opening CSF pressure, the possibility of IIH as a confounding factor in the control group is expected to be very minimal.

Use of patients without clinical or radiologic abnormalities is not optimal for the healthy group and is another limitation of this study. However, nonrefractory headaches and lack of other clinical or radiologic abnormalities minimize the possibility of bias due to sampling issues. The coronal T2 sequences are regularly acquired as part of the routine brain MR imaging examination in our institution. Some other imaging centers may not include the coronal T2-weighted sequences in their routine brain MR imaging protocols. On the basis of our experience, the measurement values on the coronal T2 sequences were comparable with those on the coronal postcontrast sequences in most subjects, considering that enhancement of the cisternal walls of the Meckel cave facilitates depiction of the borders of the MC.

Finally, asymmetric enlargement of the MC has also been reported in the literature in patients with a diagnosis of cutaneous hemangioma-vascular complex syndrome (also known as PHACE syndrome) and other sellar abnormalities, which could be a potential distractor in the diagnosis of IIH. Therefore, enlargement of the MC structures is by no means specific to elevated intracranial pressure and may also appear in other pathologies.

\section{CONCLUSIONS}

Our study confirms the shape and size of the Meckel cave as noninvasive diagnostic imaging markers, which are both relatively sensitive and specific for the diagnosis of IIH.

Disclosures: Refaat E. Gabr-UNRELATED: Employment: Department of Diagnostic Radiology, University of Texas at Houston, Houston, Texas.

AJNR Am J Neuroradiol 41:1487-94 Aug 2020 www.ajnr.org 


\section{REFERENCES}

1. McCluskey G, Doherty-Allan R, McCarron P, et al. Meta-analysis and systematic review of population-based epidemiological studies in idiopathic intracranial hypertension. Eur J Neurol 2018;25:121827 CrossRef Medline

2. Mollan SP, Aguiar M, Evison F, et al. The expanding burden of idiopathic intracranial hypertension. Eye (Lond) 2019;33:478-85 CrossRef Medline

3. Friedman DI, Jacobson DM. Diagnostic criteria for idiopathic intracranial hypertension. Neurology 2002;59:1492-95 CrossRef Medline

4. Friedman DI, Liu GT, Digre KB. Revised diagnostic criteria for the pseudotumor cerebri syndrome in adults and children. Neurology 2013;81:1159-65 CrossRef Medline

5. Smith JL. Whence pseudotumor cerebri? J Clin Neuroophthalmol 1985;5:55-56 Medline

6. Brodsky MC, Vaphiades M. Magnetic resonance imaging in pseudotumor cerebri. Ophthalmology 1998;105:1686-93 CrossRef Medline

7. Mallery RM, Rehmani OF, Woo JH, et al. Utility of magnetic resonance imaging features for improving the diagnosis of idiopathic intracranial hypertension without papilledema. J Neuroophthalmol 2019;39:299-307 CrossRef Medline

8. Bialer OY, Rueda MP, Bruce BB, et al. Meningoceles in idiopathic intracranial hypertension. AJR Am J Roentgenol 2014;202:608-13 CrossRef Medline

9. Aaron GP, Illing E, Lambertsen Z, et al. Enlargement of Meckel's cave in patients with spontaneous cerebrospinal fluid leaks. Int Forum Allergy Rhinol 2017;7:421-24 CrossRef Medline

10. Degnan AJ, Levy LM. Narrowing of Meckel's cave and cavernous sinus and enlargement of the optic nerve sheath in pseudotumor cerebri. J Comput Assist Tomogr 2011;35:308-12 CrossRef Medline

11. Morris PP, Black DF, Port J, et al. Transverse sinus stenosis is the most sensitive MR imaging correlate of idiopathic intracranial hypertension. AJNR Am J Neuroradiol 2017;38:471-77 CrossRef Medline

12. Bidot S, Saindane AM, Peragallo JH, et al. Brain imaging in idiopathic intracranial hypertension. J Neuroophthalmol 2015;35:400-11 CrossRef Medline

13. Kahle KT, Kulkarni AV, Limbrick DD, et al. Hydrocephalus in children. Lancet 2016;387:788-99 CrossRef Medline

14. Plog BA, Nedergaard $M$. The glymphatic system in central nervous system health and disease: past, present, and future. Annu Rev Pathol 2018;13:379-94 CrossRef Medline

15. Rasmussen $M K$, Mestre $H$, Nedergaard $M$. The glymphatic pathway in neurological disorders. Lancet Neurol 2018;17:1016-24 CrossRef

16. Portelli M, Papageorgiou PN. An update on idiopathic intracranial hypertension. Acta Neurochir (Wien) 2017;159:491-99 CrossRef Medline

17. Markey KA, Mollan SP, Jensen RH, et al. Understanding idiopathic intracranial hypertension: mechanisms, management, and future directions. Lancet Neurol 2016;15:78-91 CrossRef Medline 